\title{
Effect of Amino Acids on the Generation of Ginsenoside Rg3 Epimers by Heat Processing and the Anticancer Activities of Epimers in A2780 Human Ovarian Cancer Cells
}

\author{
Jun Yeon Park, ${ }^{1}$ Pilju Choi, ${ }^{2}$ Dahae Lee, ${ }^{1}$ Taejung Kim, ${ }^{2}$ Eun Bee Jung, ${ }^{1}$ \\ Buyng-Su Hwang, ${ }^{2}$ Ki Sung Kang, ${ }^{1}$ and Jungyeob Ham ${ }^{2}$ \\ ${ }^{1}$ College of Korean Medicine, Gachon University, Seongnam 461-701, Republic of Korea \\ ${ }^{2}$ KIST Gangneung Institute of Natural Products, Korea Institute of Science and Technology, Gangneung 210-340, Republic of Korea
}

Correspondence should be addressed to Ki Sung Kang; kkang@gachon.ac.kr and Jungyeob Ham; ham0606@kist.re.kr

Received 26 August 2015; Revised 13 November 2015; Accepted 3 December 2015

Academic Editor: Man Hee Rhee

Copyright ( 2016 Jun Yeon Park et al. This is an open access article distributed under the Creative Commons Attribution License, which permits unrestricted use, distribution, and reproduction in any medium, provided the original work is properly cited.

Ginsenosides are the active components of Panax ginseng. Many research studies indicate that these deglycosylated, less-polar ginsenosides have better bioactivity than the major ginsenosides. In the present study, we sought to verify the enhanced anticancer effect of $P$. ginseng extract after undergoing the Maillard reaction as well as elucidate the underlying mechanism of action. The effects of 9 amino acids were tested; among them, the content of 20(S)-Rg3 in the ginseng extract increased to more than 30, 20, and $20 \%$ when processed with valine, arginine, and alanine, respectively, compared with that after normal heat processing. The ginseng extract that was heat-processed with arginine exhibited the most potent inhibitory effect on A2780 ovarian cancer cell proliferation. Therefore, the generation of 20 (S)-Rg3 was suggested to be involved in this effect. Moreover, the inhibitory effect of 20(S)-Rg3 on A2780 cell proliferation was significantly stronger than that of 20(R)-Rg3. Protein expression levels of cleaved caspase3, caspase-8, caspase-9, and PARP in the A2780 ovarian cancer cells markedly increased, whereas the expression of BID decreased after 20(S)-Rg3 treatment. Therefore, we confirmed that the anticancer effects of the products of ginseng that was heat-processed with arginine are mediated mainly via the generation of the less-polar ginsenoside 20(S)-Rg3.

\section{Introduction}

The use of naturally occurring compounds for the design of novel anticancer drugs has increased in recent years, especially owing to increasing demand for natural remedies among cancer patients [1-4]. Experimental as well as clinical studies have shown that ginseng and its components are beneficial for chemotherapy since they inhibit proliferation and induce apoptosis in various malignancies, including ovarian cancer [5-8].

The roots of ginseng contain dammarane-based saponins, which are composed of 1 to 4 glycosyl moieties combined with a dammarane backbone. The saponins that are present in high concentrations in ginseng are the major ginsenosides $\mathrm{Rb} 1, \mathrm{Rb} 2, \mathrm{Rc}, \mathrm{Rd}$, and Re (Figure 1) [9, 10]. In addition, lesspolar ginsenosides such as $\operatorname{Rg} 3$ and $\operatorname{Rg} 5$ can be obtained from the deglycosylation of the major ginsenosides [11].
Many research studies indicate that these deglycosylated, lesspolar ginsenosides have better bioactivity than the major ginsenosides [5, 12, 13]. Ginsenoside Rg3 activated caspase-3 and Bax protein in human hepatocellular carcinoma cells [14]. Ginsenoside Rg3 also suppressed lung cancer migration and invasion by inhibiting the transforming growth factor-beta 1induced epithelial-mesenchymal transition [15]. Ginsenoside Rg5 blocked cell cycle of hepatocellular carcinoma cells at the Gl/S transition phase by downregulating cyclin E-dependent kinase activity [16]. Ginsenoside Rk1 induces apoptosis, G1 phase arrest, and autophagy in SK-HEP-1 cells [17, 18]. Therefore, the development of ginseng extract containing an increased concentration of deglycosylated ginsenosides has been investigated.

Heat processing and enzyme-based methods are two major techniques that can achieve deglycosylation of ginsenosides [9]. The Maillard reaction of amino acids with 


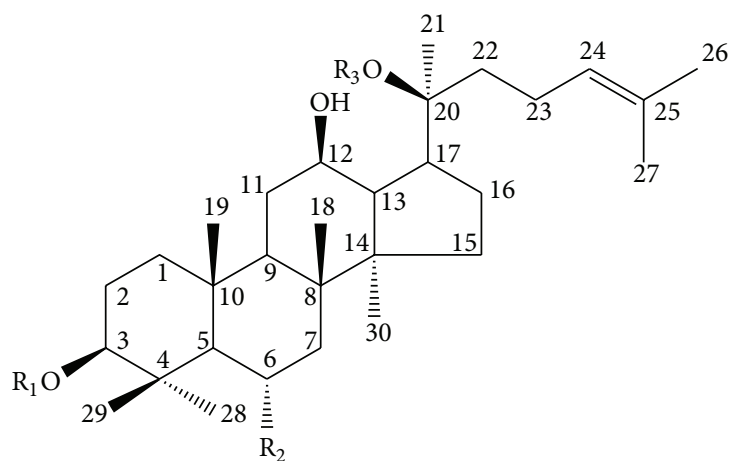

Rb1: $\mathrm{R}_{1}=\mathrm{Glc}(2 \leftarrow 1) \mathrm{Glc}, \mathrm{R}_{2}=\mathrm{H}, \mathrm{R}_{3}=\mathrm{Glc}(6 \leftarrow 1) \mathrm{Glc}$

$\mathrm{Rb} 2: \mathrm{R}_{1}=\mathrm{Glc}(2 \leftarrow 1) \mathrm{Glc}, \mathrm{R}_{2}=\mathrm{H}, \mathrm{R}_{3}=\mathrm{Glc}(6 \leftarrow 1) \operatorname{Ara}(\mathrm{p})$

Rc: $\mathrm{R}_{1}=\mathrm{Glc}(2 \leftarrow 1) \mathrm{Glc}, \mathrm{R}_{2}=\mathrm{H}, \mathrm{R}_{3}=\mathrm{Glc}(6 \leftarrow 1) \operatorname{Ara}(\mathrm{f})$

Rd: $\mathrm{R}_{1}=\mathrm{Glc}(2 \leftarrow 1) \mathrm{Glc}, \mathrm{R}_{2}=\mathrm{H}, \mathrm{R}_{3}=\mathrm{Glc}$

Re: $\mathrm{R}_{1}=\mathrm{H}, \mathrm{R}_{2}=\mathrm{O}-\mathrm{Glc}(2 \leftarrow 1) \mathrm{Rha}, \mathrm{R}_{3}=\mathrm{Glc}$

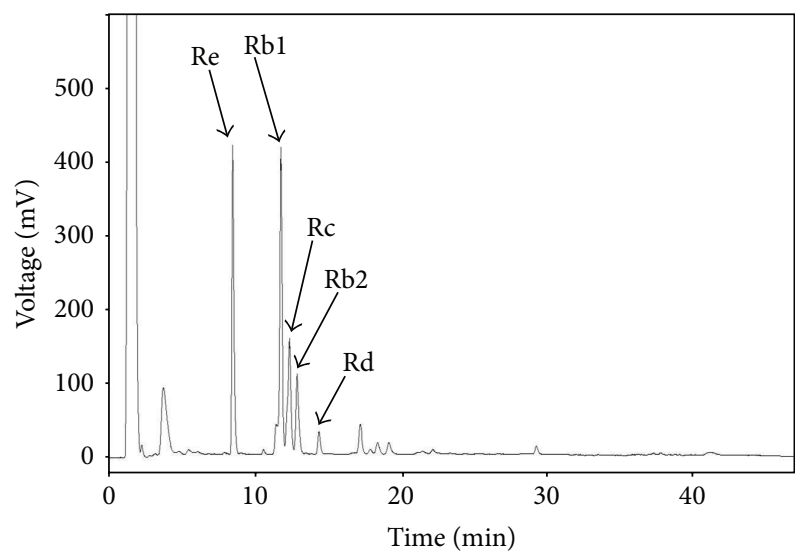

(b)

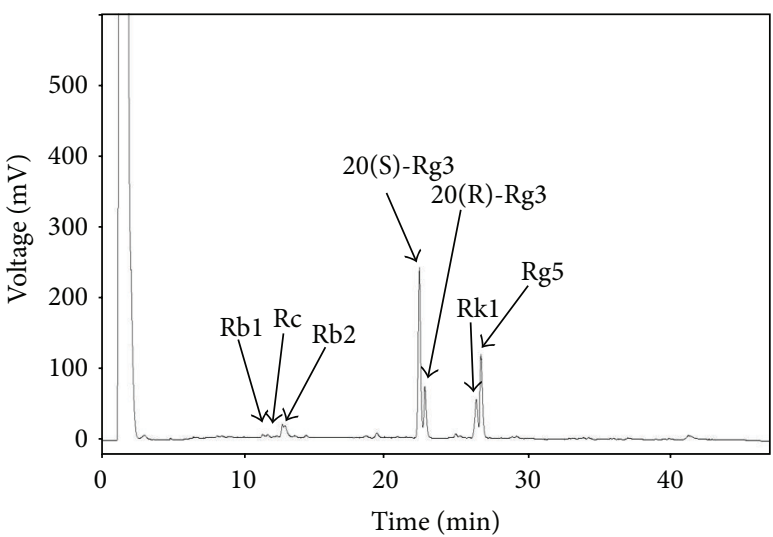

(d)

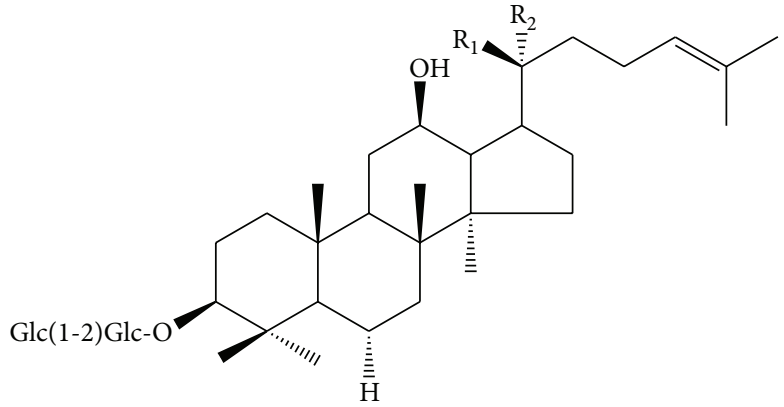

20(R)-Rg3: $\mathrm{R}_{1}=-\mathrm{CH}_{3}, \mathrm{R}_{2}=-\mathrm{OH}$

20(S)-Rg3: $\mathrm{R}_{1}=-\mathrm{OH}, \mathrm{R}_{2}=-\mathrm{CH}_{3}$

(a)

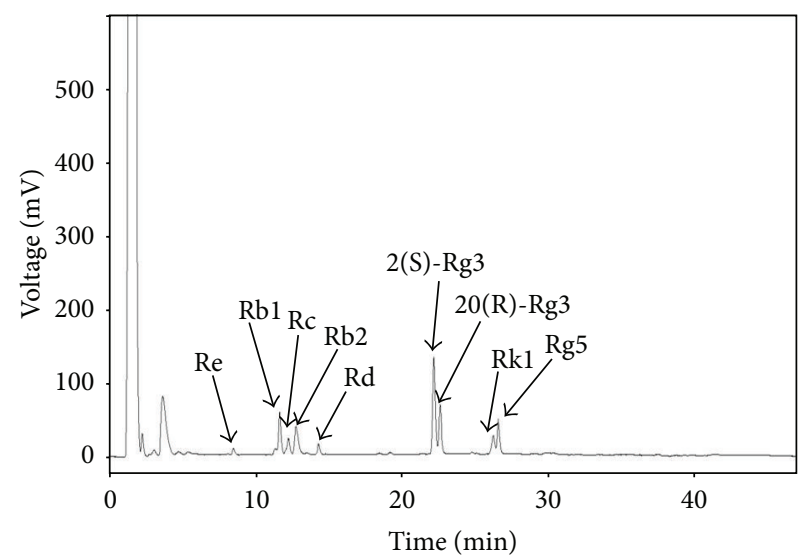

(c)

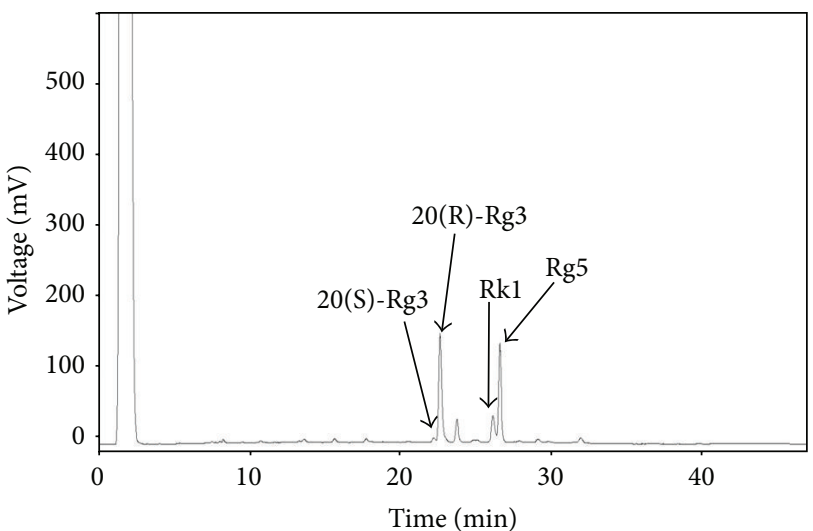

(e)

Figure 1: Changes in the concentrations of ginsenosides in Panax ginseng extract after heat processing with amino acids. (a) Structures of major and deglycosylated ginsenosides. (b) HPL chromatogram of Panax ginseng extract before heat processing. (c) HPL chromatogram of Panax ginseng extract after heat processing. (d) HPL chromatogram of Panax ginseng extract after heat processing with arginine. (e) HPL chromatogram of Panax ginseng extract after heat processing with leucine. -Glc: D-glucopyranosyl, -Rha: L-rhamnopyranosyl, -Ara(f): Larabinofuranosyl, and -Ara(p): L-arabinopyranosyl. 
sugars is a nonenzymatic browning reaction that takes place during the processing, cooking, and storage of foods. It is well known that Maillard reaction products produced in red ginseng have antioxidant activity $[19,20]$. We have extensively studied and developed novel heat processing methods that facilitate the deglycosylation of major ginsenosides Rb1, Rb2, $\mathrm{Rc}, \mathrm{Rd}$, and $\mathrm{Re}[4,10]$. Recently, we identified the importance of the Maillard reaction in the generation of less-polar ginsenosides with a protective effect in the kidney [21]. In the present study, we sought to further improve the anticancer effect of Panax ginseng extract via the Maillard reaction as well as elucidate its mechanism of action.

\section{Materials and Methods}

2.1. Chemicals and Reagents. Ginsenoside standards Rb1, Rb2, Rc, Rd, Re, 20(S)-Rg3, 20(R)-Rg3, Rk1, and Rg5 were purchased from Ambo Institute (Seoul, Korea). L-Amino acid standards alanine, arginine, glutamine, glycine, leucine, lysine, serine, trans-4-hydroxy-L-proline, and valine were purchased from Sigma Aldrich (Saint Louis, MO, USA). Monoclonal antibodies against cleaved caspase- 8 and $\beta$-actin and polyclonal antibodies against cleaved caspase- 3 , cleaved caspase-9, BH3-interacting domain death (BID) agonist, and poly (ADP-ribose) polymerase (PARP) were purchased from Cell Signaling Technology, Inc. (Danvers, MA, USA).

\subsection{Maillard Reaction Model Experiment Using Ginseng} Extract with Amino Acids. Four-year-old ginseng (P.ginseng) was purchased from a local ginseng market in Seoul (Korea). Dried ginseng was ground until it could pass through an 80 -mesh sieve. It was then extracted under reflux with $50 \%$ $\mathrm{EtOH}$ three times at $70^{\circ} \mathrm{C}$ for $2 \mathrm{~h}$ and filtered through filter paper (Advantec, Tokyo, Japan). The solvent was evaporated in vacuo to give a $50 \% \mathrm{EtOH}$ extract with a yield of about $20 \%$, by weight, of the original ginseng powder. The heat processing conditions were designed such that the total ginsenosides contained in the ginseng extract reacted with the same molar concentrations of amino acids. The mixtures were steamed together at $120^{\circ} \mathrm{C}$ for $3 \mathrm{~h}$ as reported previously [22]. After drying at $50^{\circ} \mathrm{C}$ for 3 days, the untreated and heat-processed ginseng extracts with amino acids were prepared.

2.3. Antiproliferative Effect on A2780 Ovarian Cancer Cells. The human ovarian cancer A2780 cell line was purchased from the American Type Culture Collection (ATCC, Manassas, VA). The cells were grown in RPMI1640 medium (Cellgro, Manassas, VA) supplemented with $10 \%$ fetal bovine serum (Gibco BRL, Carlsbad, MD), 100 units/mL penicillin, and $100 \mu \mathrm{g} / \mathrm{mL}$ streptomycin. They were incubated at $37^{\circ} \mathrm{C}$ in a humidified atmosphere with $5 \% \mathrm{CO}_{2}$. The Cell Counting Kit-8 (CCK-8, Dojindo Laboratories, Japan) was used to determine cell proliferation according to the manufacturer's recommendations. Briefly, cells were seeded in 96-well plates at a density of $1 \times 10^{4}$ cells/well and incubated for $24 \mathrm{~h}$ at $37^{\circ} \mathrm{C}$. The cells were treated with different concentrations of compounds. After incubation for $24 \mathrm{~h}, 10 \mu \mathrm{L}$ of the kit reagent was added to each well, and the cells were incubated for an additional hour. Cell proliferation was measured by scanning with a microplate reader at $450 \mathrm{~nm}$. Control cells were exposed to culture media containing $0.5 \% \mathrm{v} / \mathrm{v}$ dimethyl sulfoxide (DMSO).

2.4. Analysis and Structural Confirmation of Ginsenosides. The analytical reversed-phase high-performance liquid chromatography (HPLC) system comprised a solvent degasser (Agilent, G1322A), binary pump (Agilent, G1312C), an autosampler (Agilent, G1329B), and model ZAM 3000 Evaporative Light Scattering Detector (ELSD) (Young Lin, South Korea). ELSD conditions were optimized in order to achieve maximum sensitivity; the temperature of the nebulizer was set for $50^{\circ} \mathrm{C}$, and $\mathrm{N}_{2}$ was used as the nebulizing gas at a pressure of 2.0 bar. A Phenomenex Luna C18 column (150 $\times 4.6 \mathrm{~mm}, 5 \mu \mathrm{m})$ was used, and the mobile phase comprised binary gradient of solvent $\mathrm{A}$ (acetonitrile: water : $5 \%$ acetic acid in water $=15: 80: 5$ ) and solvent $B$ (acetonitrile $:$ water $=80: 20$ ) at a flow rate of $1.0 \mathrm{~mL} / \mathrm{min}$. The gradient flow program was as follows: initial $0 \% \mathrm{~B} ; 6 \mathrm{~min}, 30 \% \mathrm{~B} ; 18 \mathrm{~min}$, $50 \% \mathrm{~B} ; 30 \mathrm{~min}, 100 \% \mathrm{~B} ; 37 \mathrm{~min}, 100 \% \mathrm{~B} ; 42 \mathrm{~min}, 0 \% \mathrm{~B}$.

2.5. Flow Cytometric Assay. To detect changes in cell cycle distribution, the treated cells were collected, washed with cold PBS, and fixed in $70 \%$ ethanol at $4^{\circ} \mathrm{C}$ for $30 \mathrm{~min}$. The cells were then washed twice with PBS and resuspended in $500 \mu \mathrm{L}$ of a propidium iodide (PI) staining solution containing $40 \mu \mathrm{g} / \mathrm{mL}$ PI and $20 \mu \mathrm{g} / \mathrm{mL}$ RNase A in PBS. Then, the cells were incubated at room temperature for $30 \mathrm{~min}$ in the dark and analyzed with a FACSCalibur flow cytometer (BectonDickinson, San Jose, CA, USA) and the ModFit LT version 2.0 computer program.

2.6. Western Blotting Analysis. Cells $\left(8 \times 10^{5}\right.$ cells $)$ grown in $60 \mathrm{~mm}$ dishes were treated with the indicated concentration of samples for $24 \mathrm{~h}$. Whole-cell extracts were then prepared according to the manufacturer's instructions using RIPA buffer (Cell Signaling, MA, USA) supplemented with $1 \mathrm{x}$ protease inhibitor cocktail and $1 \mathrm{mM}$ phenylmethylsulfonyl fluoride (PMSF) [23]. Proteins (whole-cell extracts, $30 \mu \mathrm{g} /$ lane) were separated by electrophoresis in a precast 415\% Mini-PROTEAN TGX gel (Bio-Rad, CA, USA), blotted onto PVDF transfer membranes, and analyzed with epitopespecific primary and secondary antibodies. Bound antibodies were visualized using ECL Advance Western Blotting Detection Reagents (GE Healthcare, UK) and a LAS 4000 imaging system (Fujifilm, Japan).

2.7. Statistical Analysis. Statistical significance was determined through analysis of variance (ANOVA) followed by a multiple comparison test with a Bonferroni adjustment. $P$ values of less than 0.05 were considered statistically significant.

\section{Results and Discussion}

In the present study, we developed a novel method of converting ginsenosides, the major active components of 
TABLE 1: Changes in contents of ginsenosides ( $\mu \mathrm{g} / \mathrm{mg})$.

\begin{tabular}{|c|c|c|c|c|c|c|c|c|c|}
\hline Ginsenosides & $\operatorname{Re}$ & $\mathrm{Rb} 1$ & Rc & $\mathrm{Rb} 2$ & $\mathrm{Rd}$ & $20(\mathrm{~S})-\operatorname{Rg} 3$ & $20(\mathrm{R})-\mathrm{Rg} 3$ & $\mathrm{Rk1}$ & Rg5 \\
\hline Nontreated & 26.1 & 35.0 & 15.1 & 19.7 & 4.1 & - & - & - & - \\
\hline Heat & 2.0 & 1.4 & 7.8 & 3.4 & 12.1 & 31.6 & 20.5 & 7.3 & 13.9 \\
\hline Heat/glutamine & - & - & - & - & - & 7.4 & 30.4 & 14.1 & 48.1 \\
\hline Heat/leucine & - & - & - & - & - & 1.8 & 42.7 & 11.7 & 35.1 \\
\hline Heat/valine & 4.5 & 5.1 & 2.0 & 8.9 & 1.0 & 41.8 & 10.3 & 8.5 & 18.0 \\
\hline Heat/arginine & - & 10.6 & 0.4 & 7.7 & - & 38.5 & 11.0 & 10.4 & 21.3 \\
\hline Heat/alanine & 3.7 & 5.5 & 2.3 & 9.4 & - & 39.0 & 12.7 & 8.2 & 18.0 \\
\hline
\end{tabular}

ginseng, and enhancing the anticancer effect of ginseng by high-temperature amino acid processing.

$P$. ginseng contains a high concentration of ginsenosides. Figure 1(a) shows the structure of the major ginsenosides contained in ginseng, including Rb1, Rb2, Rc, Rd, Rg3, and $\mathrm{Re}$. The typical ginsenosides in raw ginseng include $\mathrm{Re}$, Rb1, Rc, Rb2, and Rd (Figure 1(b)). As shown in Figure 1(c), when Rb1, Rb2, Rc, Rd, Rg1, and Re are heat-processed, glucose, which is a glycoside located at position 20, may be dissociated and subsequent dehydration may occur at position 20, such that ginsenosides Rb1, Rb2, Rc, Rd, Rg1, and Re are converted into Rg3, Rg5, and Rk1 (compare Figures 1(b) and 1(c)). In the present study, we further examined the effects of amino acids on the generation of these less-polar ginsenosides. The effects of 9 amino acids (alanine, arginine, glutamine, glycine, leucine, lysine, serine, trans-4-hydroxyL-proline, and valine) were tested; among them, 5 amino acids (glutamine, leucine, valine, arginine, and alanine) had a significant influence on the generation of less-polar ginsenosides during heat processing. The concentration of $20(\mathrm{R})-\mathrm{Rg} 3$ in the ginseng extract increased to more than 50 and $100 \%$ when processed with glutamine and leucine, respectively, compared to 20(R)-Rg3 concentration after normal heat processing (Table 1). In addition, the contents of the ginsenoside 20(S)-Rg3 decreased in the ginseng extract, while those of ginsenosides Rk1 and Rg5 increased when the extract was heat-processed with glutamine or leucine. Interestingly, the concentration of $20(\mathrm{~S})-\mathrm{Rg} 3$ in the ginseng extract increased to more than 30,20 , and $20 \%$ when it is processed with valine, arginine, and alanine, respectively, compared to that obtained after normal heat processing (Table 1 ). In addition, the concentration of ginsenoside 20(R)-Rg3 decreased, while those of ginsenosides $\mathrm{Rk} 1$ and $\mathrm{Rg} 5$ increased in the extract when it was heat-processed with valine, arginine, and alanine. Therefore, certain amino acids were found to affect the generation of less-polar ginsenosides. Among them, two representative amino acids, leucine and arginine, were selected for the evaluation of anticancer effects. Figures 1(d) and 1(e) show the representative HPL chromatogram of the Panax ginseng extract after heat processing with arginine and leucine. These heat processing conditions were selected because they generated the greatest total amount of less-polar ginsenosides and were stereospecific in their generation of Rg3 epimers (Table 1, Figure 1).

The percent viability of A2780 ovarian cancer cells after treatment with ginseng extract (with leucine or arginine) and compounds (20(R)-Rg3 and 20(S)-Rg3) is shown in Figure 2. In Figure 2(a), each ginseng extract inhibited A2780 cell proliferation in a dose-dependent manner. Among them, the ginseng extract that was heat-processed with arginine exhibited the most potent inhibition. Therefore, the generation of 20 (S)-Rg3 was suggested to be involved in the increased anticancer effect. The effect of 20(R)-Rg3 and 20(S)-Rg3 on the viability of A2780 is shown in Figure 2(b). It can be seen that 20(R)-Rg3 and 20(S)-Rg3 inhibited A2780 cell proliferation in a dose-dependent manner, and the inhibitory effect of 20 (S)-Rg3 was significantly stronger than that of $20(\mathrm{R})-\mathrm{Rg} 3$. The effect of 20(S)-Rg3 on the cell cycle distribution at $24 \mathrm{~h}$ was tested using flow cytometric assay. However, treatment with 20(S)-Rg3 did not affect A2780 cancer cell cycle arrest (data not shown). Therefore, it might be reasonable to identify apoptosis as a mechanistic pathway of heat-processed ginseng extract with arginine or $20(\mathrm{~S})-\mathrm{Rg} 3$.

Dysregulation of apoptosis leads to various human pathologies including cancer $[24,25]$. Intrinsic apoptosis is induced through the activation of specific signaling pathways, while extrinsic apoptosis is initiated through transmembrane death receptors $[26,27]$. Initiation and execution of these processes are regulated by initiating factors, including proteases such as caspases and the $\mathrm{Bcl}-2$ family of proteins [28]. Caspase- 8 may in turn either directly cleave effector caspases, such as caspase-3, or amplify the death signal through the translocation of Bcl-2 family member BID to the mitochondria to activate the downstream components of the mitochondria apoptotic pathway $[29,30]$. The cleavage of PARP is also an important indicator of caspase- 3 activation during apoptosis [31].

Figure 3 shows the protein expressions of cleaved caspase3 , cleaved caspase-8, cleaved caspase-9, BID, and PARP in the control and experimental groups (treatment with arginine and 20(S)-Rg3). Protein expression levels of cleaved caspase3 , caspase-8, and caspase-9 in A2780 ovarian cancer cells markedly increased after $20(\mathrm{~S})-\mathrm{Rg} 3$ treatment. In addition, a decrease in protein expression of BID and an increase in cleavage of PARP were noted after 20(S)-Rg3 treatment. Therefore, the apoptotic effects of the products of ginseng that was heat-processed with arginine were confirmed to be mainly mediated by the generation of the less-polar ginsenoside 20(S)-Rg3.

In conclusion, the modulation of the anticancer effect of a $P$. ginseng extract via Maillard reaction was verified 


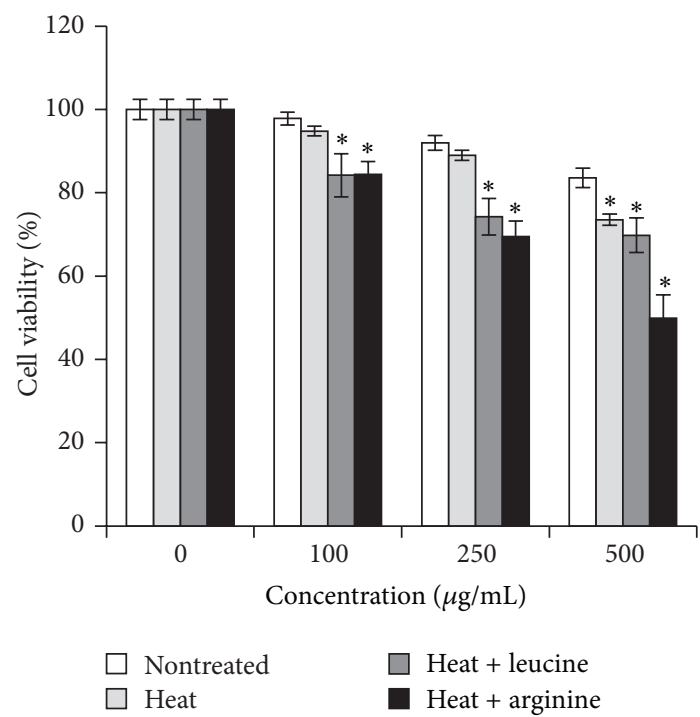

(a)

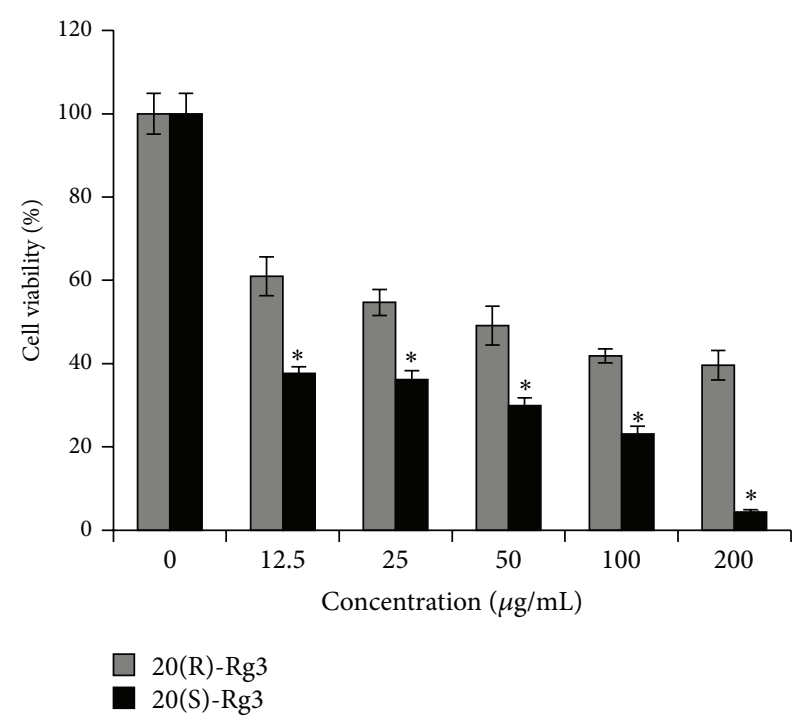

(b)

FIGURE 2: Changes in the effects of Panax ginseng extract on A2780 cell proliferation after heat processing with or without amino acids. (a) Cells were treated with nontreated or heat-processed Panax ginseng extract with or without amino acids at different concentrations (100, 250, and $500 \mu \mathrm{g} / \mathrm{mL}$ ) for $24 \mathrm{~h}$. (b) Cells were treated with ginsenoside $20(\mathrm{~S})-\mathrm{Rg} 3$ or ginsenoside $20(\mathrm{R})-\mathrm{Rg} 3$ at different concentrations (12.5, 25, 50,100 , and $200 \mu \mathrm{g} / \mathrm{mL}$ ) for $24 \mathrm{~h}$. Relative cell proliferation was measured by the CCK- 8 assay. Each value represents the mean \pm SD of three independent experiments. ${ }^{*} P<0.05$ compared with nontreated value.

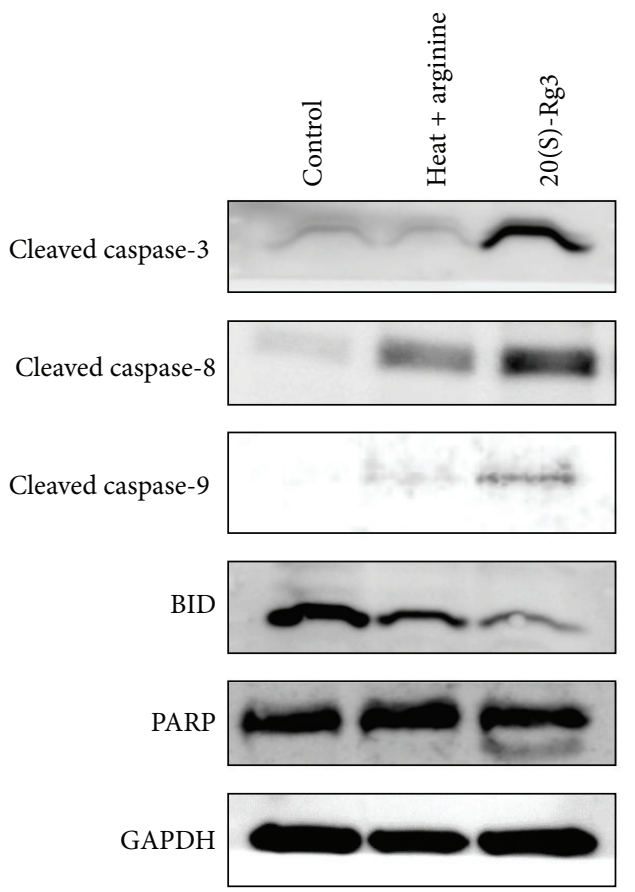

FIGURE 3: Effects of Panax ginseng extract heat-processed with arginine and ginsenoside 20(S)-Rg3 on apoptosis in A2780 cells. Western blotting results showing the levels of cleaved caspase-3, cleaved caspase-8, cleaved caspase-9, BID, and PARP in A2780 cells treated with heat-processed Panax ginseng extract with arginine $(500 \mu \mathrm{g} / \mathrm{mL})$ and ginsenoside 20(S)-Rg3 $(12.5 \mu \mathrm{g} / \mathrm{mL})$ for $24 \mathrm{~h}$. Thirty micrograms of each protein was separated by SDS-PAGE. GAPDH was used as an internal control. and its mechanism of action was further outlined. The heatprocessed ginseng had abundance of ginsenosides Rg3, Rg5, and Rk1. After treatment with amino acids, the ginseng had a higher concentration of 20 (S)-Rg3 and 20(R)-Rg3 and thus had an increased medicinal effect. We demonstrated that 20(S)-Rg3 suppressed the growth of A2780 ovarian cancer cells in vitro. Therefore, heat processing by Maillard reaction is a useful method to enhance the anticancer effect of ginseng by increasing the content of 20 (S)-Rg3.

\section{Conflict of Interests}

The authors declare no conflict of interests in this work.

\section{Authors' Contribution}

Jun Yeon Park and Pilju Choi contributed equally to the content of this paper.

\section{Acknowledgments}

This work was supported by the Korea Institute of Science and Technology institutional program (2Z04390). This research was also conducted under the Industrial Infrastructure Program for Fundamental Technologies (N0000885) which is funded by the Ministry of Trade, Industry and Energy (MOTIE, Korea). 


\section{References}

[1] L. H. Hurley, "DNA and its associated processes as targets for cancer therapy," Nature Reviews Cancer, vol. 2, no. 3, pp. 188200, 2002.

[2] Y.-T. Kim, S.-K. Kim, Y.-J. Jeon, and S. J. Park, “Seahorse-derived peptide suppresses invasive migration of HT1080 fibrosarcoma cells by competing with intracellular $\alpha$-enolase for plasminogen binding and inhibiting uPA-mediated activation of plasminogen," BMB Reports, vol. 47, no. 12, pp. 691-696, 2014.

[3] N. C. Miltenburg and W. Boogerd, "Chemotherapy-induced neuropathy: a comprehensive survey," Cancer Treatment Reviews, vol. 40, no. 7, pp. 872-882, 2014.

[4] N. Yamabe, Y.-J. Kim, S. Lee et al., "Increase in antioxidant and anticancer effects of ginsenoside Re-lysine mixture by Maillard reaction," Food Chemistry, vol. 138, no. 2-3, pp. 876-883, 2013.

[5] Y.-S. Keum, K.-K. Park, J.-M. Lee et al., "Antioxidant and antitumor promoting activities of the methanol extract of heatprocessed ginseng," Cancer Letters, vol. 150, no. 1, pp. 41-48, 2000.

[6] E.-H. Park, Y.-J. Kim, N. Yamabe et al., "Stereospecific anticancer effects of ginsenoside Rg3 epimers isolated from heatprocessed american ginseng on human gastric cancer cell," Journal of Ginseng Research, vol. 38, no. 1, pp. 22-27, 2014.

[7] T. Tode, Y. Kikuchi, T. Kita, J. Hirata, E. Imaizumi, and I. Nagata, "Inhibitory effects by oral administration of ginsenoside $\mathrm{Rh}_{2}$ on the growth of human ovarian cancer cells in nude mice," Journal of Cancer Research and Clinical Oncology, vol. 120, no. 1-2, pp. 24-26, 1993.

[8] T.-K. Yun and S.-Y. Choi, "Preventive effect of ginseng intake against various human cancers: a case-control study on 1987 pairs," Cancer Epidemiology Biomarkers \& Prevention, vol. 4, no. 4, pp. 401-408, 1995.

[9] L. Jia, Y. Zhao, and X.-J. Liang, "Current evaluation of the millennium phytomedicine-ginseng (II): collected chemical entities, modern pharmacology, and clinical applications emanated from traditional chinese medicine," Current Medicinal Chemistry, vol. 16, no. 22, pp. 2924-2942, 2009.

[10] K. S. Kang, J. Ham, Y.-J. Kim, J. H. Park, E.-J. Cho, and N. Yamabe, "Heat-processed Panax ginseng and diabetic renal damage: active components and action mechanism," Journal of Ginseng Research, vol. 37, no. 4, pp. 379-388, 2013.

[11] T. H. Le, S. Y. Lee, T. R. Kim et al., "Processed Vietnamese ginseng: preliminary results in chemistry and biological activity," Journal of Ginseng Research, vol. 38, no. 2, pp. 154-159, 2014.

[12] W. Y. Kim, J. M. Kim, S. B. Han et al., "Steaming of ginseng at high temperature enhances biological activity," Journal of Natural Products, vol. 63, no. 12, pp. 1702-1704, 2000.

[13] K. S. Kang, H. Y. Kim, J. S. Pyo, and T. Yokozawa, "Increase in the free radical scavenging activity of ginseng by heat-processing," Biological \& Pharmaceutical Bulletin, vol. 29, no. 4, pp. 750-754, 2006.

[14] B.-G. Park, H.-J. Jung, Y.-W. Cho, H.-W. Lim, and C.-J. Lim, "Potentiation of antioxidative and anti-inflammatory properties of cultured wild ginseng root extract through probiotic fermentation," Journal of Pharmacy and Pharmacology, vol. 65, no. 3, pp. 457-464, 2013.

[15] Y.-J. Kim, W.-I. Choi, B.-N. Jeon et al., "Stereospecific effects of ginsenoside $20-\mathrm{Rg} 3$ inhibits TGF- $\beta 1$-induced epithelialmesenchymal transition and suppresses lung cancer migration, invasion and anoikis resistance," Toxicology, vol. 322, pp. 23-33, 2014.
[16] K. Y. Lee, Y. H. Lee, S. I. Kim, J. H. Park, and S. K. Lee, "Ginsenoside-Rg5 suppresses cyclin E-dependent protein kinase activity via up-regulating p21Cip/WAF1 and downregulating cyclin E in SK-HEP-1 cells," Anticancer Research, vol. 17, no. 2, pp. 1067-1072, 1997.

[17] Y.-J. Kim, H. C. Kwon, H. Ko et al., "Anti-tumor activity of the ginsenoside $\mathrm{Rk} 1$ in human hepatocellular carcinoma cells through inhibition of telomerase activity and induction of apoptosis," Biological and Pharmaceutical Bulletin, vol. 31, no. 5, pp. 826-830, 2008.

[18] H. Ko, Y.-J. Kim, J.-S. Park, J. H. Park, and H. O. Yang, "Autophagy inhibition enhances apoptosis induced by ginsenoside Rkl in hepatocellular carcinoma cells," Bioscience, Biotechnology and Biochemistry, vol. 73, no. 10, pp. 2183-2189, 2009.

[19] X. Li, Y. Zheng, M. Liu, and L. Zhang, "A study on maillard reaction and its products during processing of red ginseng," Zhongguo Zhong Yao Za Zhi, vol. 24, no. 5, pp. 274-278, 1999.

[20] K. S. Kang, H. Y. Kim, J. S. Pyo, and T. Yokozawa, "Increase in the free radical scavenging activity of ginseng by heat-processing," Biological and Pharmaceutical Bulletin, vol. 29, no. 4, pp. 750754, 2006.

[21] Y.-J. Kim, N. Yamabe, P. Choi, J. W. Lee, J. Ham, and K. S. Kang, "Efficient thermal deglycosylation of ginsenoside Rd and its contribution to the improved anticancer activity of ginseng," Journal of Agricultural and Food Chemistry, vol. 61, no. 38, pp. 9185-9191, 2013.

[22] J. H. Lee, W. Lee, S. Lee et al., "Important role of Maillard reaction in the protective effect of heat-processed ginsenoside Re-serine mixture against cisplatin-induced nephrotoxicity in LLC-PK1 cells," Bioorganic and Medicinal Chemistry Letters, vol. 22, no. 17, pp. 5475-5479, 2012.

[23] J. Li, K. Jiang, X. Qiu et al., "Overexpression of CXCR4 is significantly associated with cisplatin-based chemotherapy resistance and can be a prognostic factor in epithelial ovarian cancer," BMB Reports, vol. 47, no. 1, pp. 33-38, 2014.

[24] C. B. Thompson, "Apoptosis in the pathogenesis and treatment of disease," Science, vol. 267, no. 5203, pp. 1456-1462, 1995.

[25] J. Yuan and B. A. Yankner, "Apoptosis in the nervous system," Nature, vol. 407, no. 6805, pp. 802-809, 2000.

[26] M. Brentnall, L. Rodriguez-Menocal, R. L. de Guevara, E. Cepero, and L. H. Boise, "Caspase-9, caspase-3 and caspase-7 have distinct roles during intrinsic apoptosis," BMC Cell Biology, vol. 14, article 32, 2013.

[27] N. N. Danial and S. J. Korsmeyer, "Cell death: critical control points," Cell, vol. 116, no. 2, pp. 205-219, 2004.

[28] L. Galluzzi, I. Vitale, J. M. Abrams et al., "Molecular definitions of cell death subroutines: recommendations of the Nomenclature Committee on Cell Death 2012," Cell Death and Differentiation, vol. 19, no. 1, pp. 107-120, 2012.

[29] D. R. Green, "Apoptotic pathways: the roads to ruin," Cell, vol. 94, no. 6, pp. 695-698, 1998.

[30] A. Hartmann, J.-D. Troadec, S. Hunot et al., "Caspase-8 is an effector in apoptotic death of dopaminergic neurons in Parkinson's disease, but pathway inhibition results in neuronal necrosis," Journal of Neuroscience, vol. 21, no. 7, pp. 2247-2255, 2001.

[31] M. Tewari, L. T. Quan, K. O’Rourke et al., "Yama/CPP32 $\beta$, a mammalian homolog of CED-3, is a CrmA-inhibitable protease that cleaves the death substrate poly(ADP-ribose) polymerase," Cell, vol. 81, no. 5, pp. 801-809, 1995. 


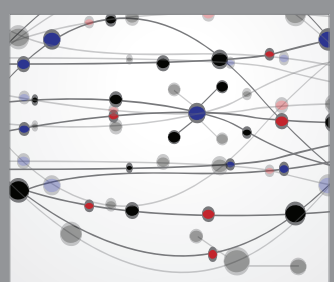

The Scientific World Journal
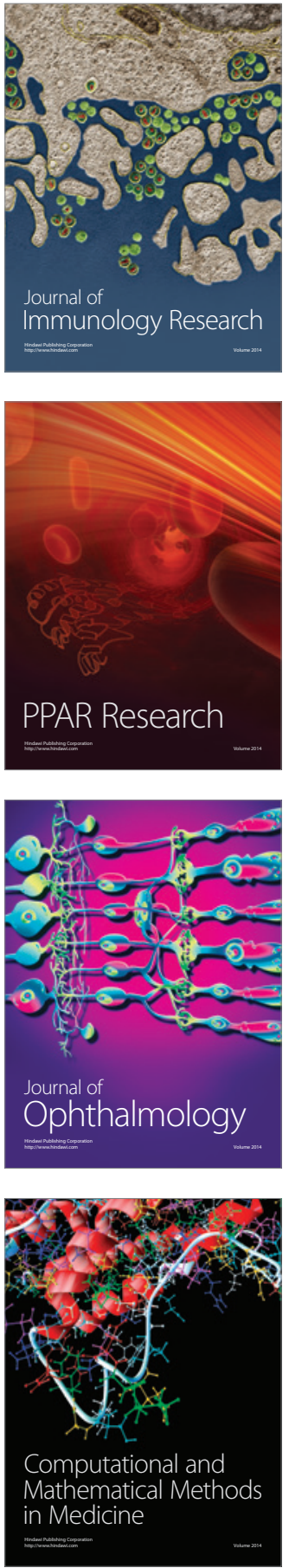

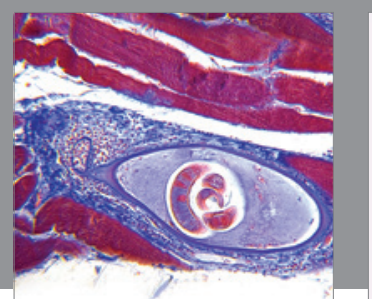

Gastroenterology Research and Practice

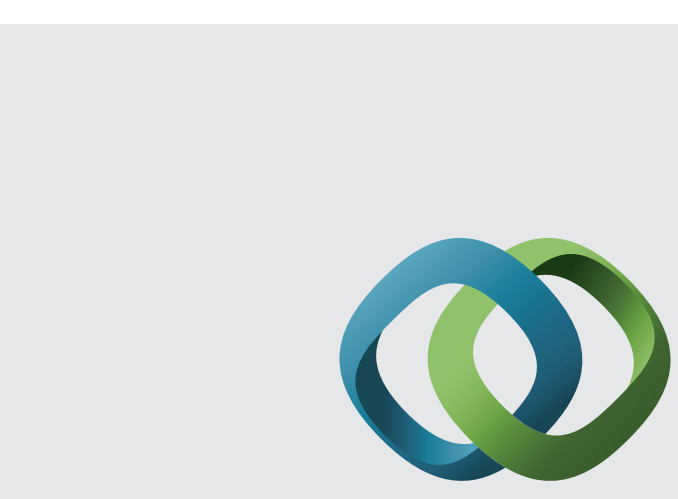

\section{Hindawi}

Submit your manuscripts at

http://www.hindawi.com
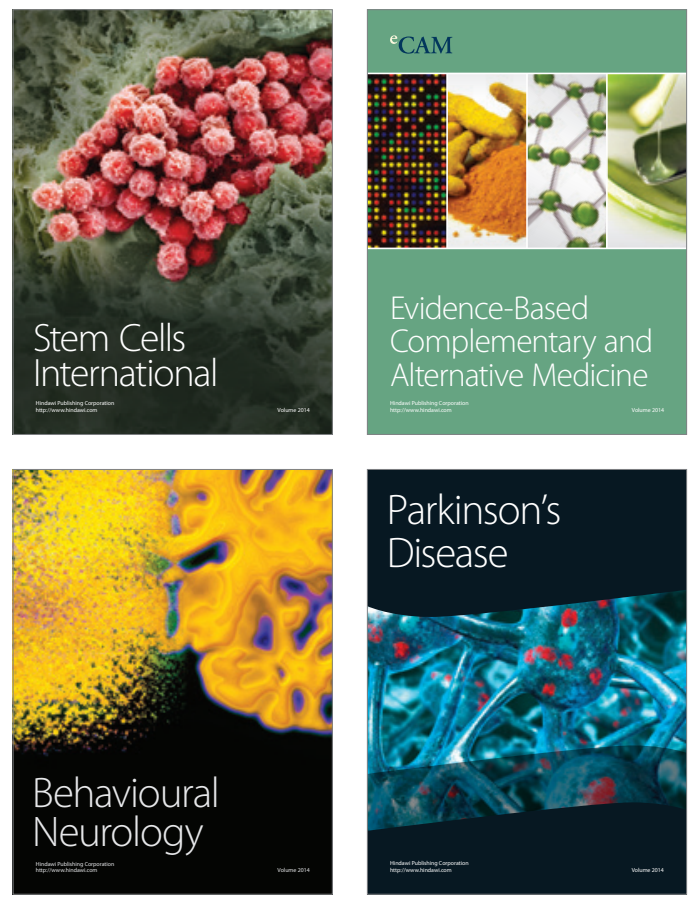
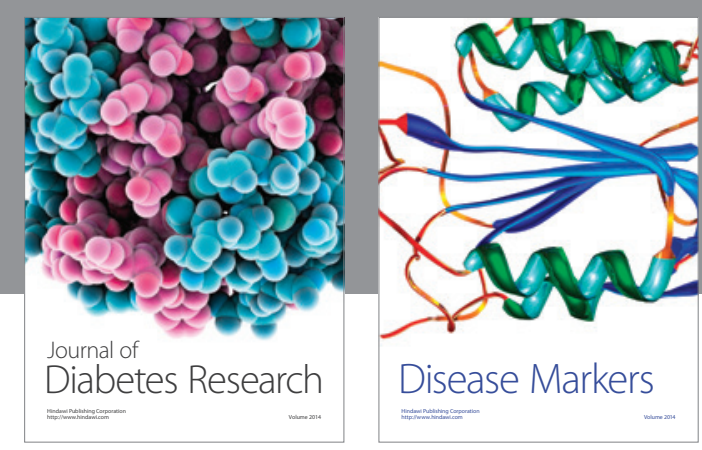

Disease Markers
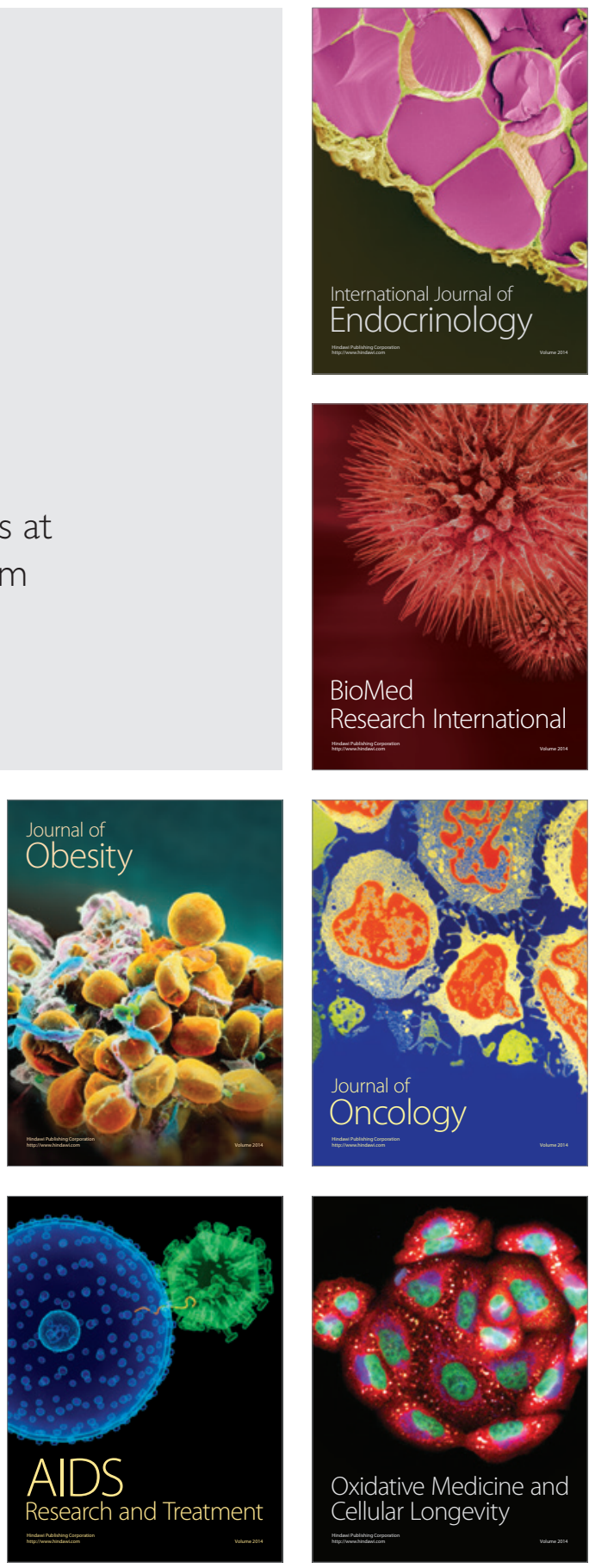